\title{
Characterization of Supercontinuum and Ultraviolet Pulses by Using XFROG
}

\author{
Baatarchuluun Tsermaa, Byung Kwan Yang, Kim Myung-Whun, and Jin Seung Kim* \\ Institute of Photonics and Information Technology, Chonbuk National University, Jeonju 561-756, Korea
}

(Received February 4, 2009 : revised March 3, 2009 : accepted March 4, 2009)

\begin{abstract}
We present cross-correlation frequency-resolved optical gating (XFROG) measurements of supercontinuum pulses generated by using a photonic crystal fiber (PCF), and ultraviolet (UV) pulses generated by frequency doubling of infrared ultra-short pulses. Since supercontinuum pulses have broad spectra, XFROG measurement typically requires using an extremely thin nonlinear crystal which has a thickness of sub-ten microns. Instead of using such a thin crystal, we employed a relatively thick crystal which was mounted on a galvanometer in order to achieve a phase-matching over the whole spectral bandwidth of the supercontinuum pulses by a crystal-dithering technique. Experimental results of the retrieved phase and intensity were in fair agreement with the independently measured data.
\end{abstract}

Keywords : Pulse characterization, FROG, XFROG, Supercontinuum, Group velocity dispersion

OCIS codes : (140.7090) Ultrafast lasers; (320.7100) Ultrafast measurements; (320.7090) Ultrafast lasers

\section{INTRODUCTION}

There has been rapid progress in the generation, manipulation and application of ultrashort laser pulses in recent years. Pulses of the order of a few femtoseconds are now routinely generated in labs, and are widely used in the areas of semiconductor physics, biomedical optics, chemistry, and high speed communications [1-4].

As the applications for femtosecond pulses increases, so does the need to completely characterize the intensity and phase of ultrashort pulses. Several such full characterization techniques were invented in the early 1990s, and their applications have truly revolutionized the field of ultrafast optics. Among these pulse measurement techniques, frequency-resolved optical gating (FROG) [4-7] and spectral phase interferometry for direct electricfield reconstruction (SPIDER) [8] are the two most widely used techniques.

Characterization of ultraviolet pulses by using secondharmonic generation (SHG)-based techniques, such as SHG FROG, involves some drawbacks when the pulses are too weak to yield efficient signal or when absorption and phase-matching constraints of nonlinear crystals

*Corresponding author: jin@chonbuk.ac.kr limit the useful nonlinear processes. These difficulties can be substantially reduced by employing XFROG technique, which is based on spectrally resolved crosscorrelation (sum frequency generation - SFG, difference frequency generation - DFG) of the weak unknown pulse with a fully characterized strong reference pulse as a function of the relative delay. The squared magnitude of the spectrum of the cross correlation signal yields the XFROG trace [9]. The strong reference pulse amplifies the weak unknown pulse and therefore allows characterization of quite weak pulses. Because XFROG uses SFG or DFG, the difficulties due to the absorption and dispersion of the crystal can be easily reduced.

This paper deals with the characterization of the intensity and the phase of complex, weak and UV pulses by using the SFG XFROG technique. As UV pulses, we have used second harmonic (SH) pulses which are generated by a commercially available frequency doubler that employs an 1-mm-thick BBO crystal as a SH generator. As complex and weak pulses, we generated supercontinuum pulses by using a PCF which has a core diameter of $1.7 \mu \mathrm{m}$ and a length of $4 \mathrm{~cm}$. Section II covers a brief theory of the XFROG measurement technique. In Section III, we describe the experimental setup and discuss the results of the XFROG measurements. In Section IV, we give the conclusion of this study. 


\section{BRIEF THEORY AND ALGORITHIM FOR XFROG}

The electric field of the XFROG signal beam is determined by the product of the electric fields of the reference and the test pulses.

$$
\left|E_{X F R O G}\right| \propto\left|E_{\text {test }} E_{\text {ref }}\right|
$$

Because the signal is proportional to the both pulses, the strong reference pulse can amplify the weak unknown pulse and therefore allows characterizing quite weak pulses [9]. The corresponding carrier frequency of the cross correlation signal is given by $\omega_{0}^{S F G}=\omega_{\text {test }}+\omega_{\text {ref }}$ for sum frequency generation, and by $\omega_{0}^{D F G}=\omega_{\text {test }}-\omega_{\text {ref }}$ for difference frequency generation. Different frequencies of the reference and the unknown pulses make the XFROG technique more powerful for measuring inconvenient wavelengths such as UV. Using XFROG, one can gate the UV pulse with a visible or infrared reference pulse, and either SFG or DFG process produces a suitable signal pulse to measure.

The squared magnitude of the spectrum of the crosscorrelation signal recorded as a function of delay between the reference pulse and the test pulse yields a XFROG trace or a spectrogram. For a SFG XFROG the spectrogram has the following form:

$$
I_{X P R O G}^{S F G}(\omega, \tau)=\left|\int_{-\infty}^{\infty} E_{\text {test }}(t) E_{r e f}(t-\tau) e^{-i \omega t} d t\right|^{2}
$$

Having the measured XFROG trace and the predetermined reference pulse as input data, one can retrieve the intensity and phase of the unknown test pulse by using

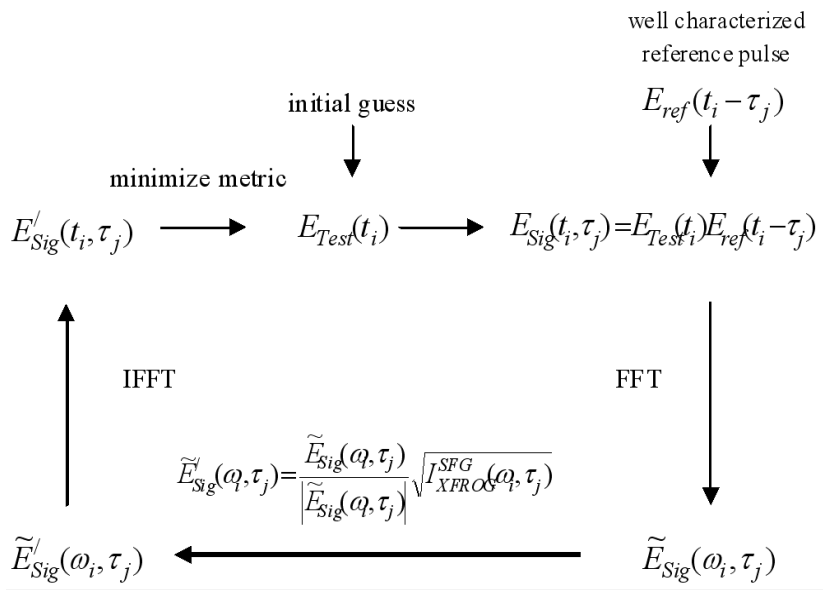

FIG. 1. Schematic of the XFROG retrieval algorithm. an iterative Fourier-transform algorithm with generalized projections [10]. The schematic of the algorithm is shown in Fig. 1. Detailed explanation for the algorithm can be found in the references $[2,7,9,10]$.

\section{EXPERIMENTAL RESULTS AND DISCUSSION}

We will give a detailed explanation of every step of our experimental setups, because it could be useful for those who are interested in characterizing the intensity and phase of complex, weak and/or UV pulses by using a XFROG technique. As we mentioned in Section I, we characterized two kinds of pulses, UV pulses and supercontinuum pulses. Taking some differences between the experimental setups into account we divided this section into two parts.

\section{UV pulse characterization}

Because of high absorption of a SHG crystal (below $190 \mathrm{~nm}$ ), it is almost impossible to use a SHG FROG technique to characterize UV pulses. But this difficulty can be reduced significantly by using a XFROG technique, since it uses cross-correlation of the unknown SH pulse and a visible or an infrared reference pulse. To show the advantage of the SFG XFROG technique, we have characterized the SH pulse, which was generated from the frequency doubler.

The arrangement of the experimental setup is shown in Fig. 2. Output pulses from the Ti:sapphire laser (788 $\mathrm{nm}, 133 \mathrm{fs}, 2.5 \mathrm{~nJ}$ ) were injected into the frequency doubler for generation of UV pulses. At the output port of the frequency doubler, the second harmonic and the residual fundamental pulses were separated, and then used as the unknown and the reference pulses for

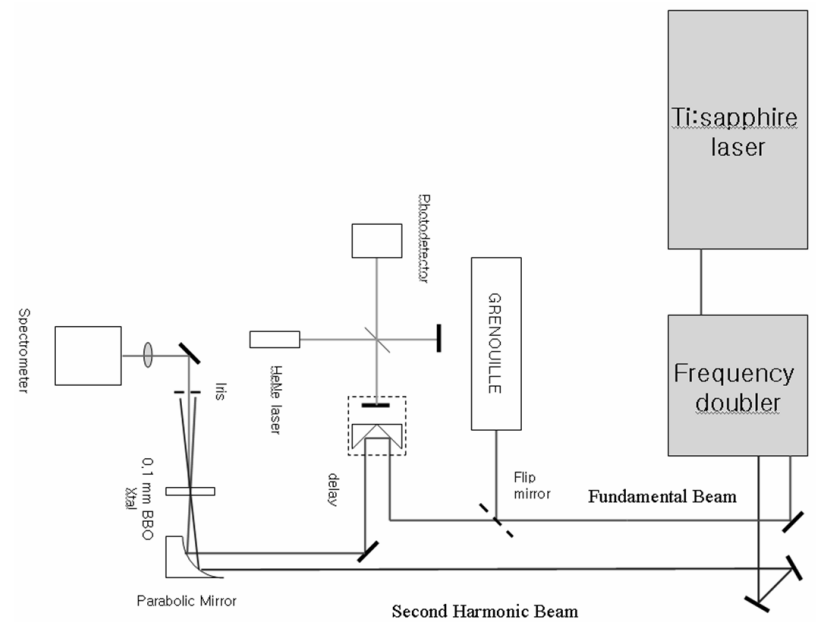

FIG. 2. Experimental setup for the UV pulse characterization by using SFG XFROG. 
the XFROG setup, respectively. The reference pulse was characterized by a simple SHG FROG apparatus named as GRENOUILLE $[11,12]$ and passed through a variable time delay. Then the two pulses were focused by an aluminum coated, parabolic mirror $(\mathrm{f}=75 \mathrm{~mm})$ onto a $100-\mu \mathrm{m}$-thick BBO crystal, and the generated sum frequency signal (around $262 \mathrm{~nm}$ ) was recorded as a function of the relative delay between the pulses with a spectrometer.

The SH pulse had an orthogonal polarization direction to the reference pulse at the output of the frequency doubler. In order to obtain an efficient sum frequency signal, we rotated the polarization direction of the SH pulse by using a half-wave plate and employed
Type I phase matching condition for generating the sum-frequency signal.

The measured intensity and phase of the reference pulse are shown in Fig. 3. The temporal width at FWHM was $133 \mathrm{fs}$ and the spectral bandwidth at FWHM of the spectrum was $6.2 \mathrm{~nm}$.

The relative delay between the UV and the reference pulses was controlled by a Michelson type interferometer with an increment of $1.77 \mathrm{fs}$. The spectrometer measured the spectrum of the generated sum frequency signal at each delay step, and all of the data are synthesized to produce a complete SFG XFROG trace. Our measured raw trace had dimensions of $64 \times 512$, and we interpolated it into a $256 \times 256$ trace for convenience of
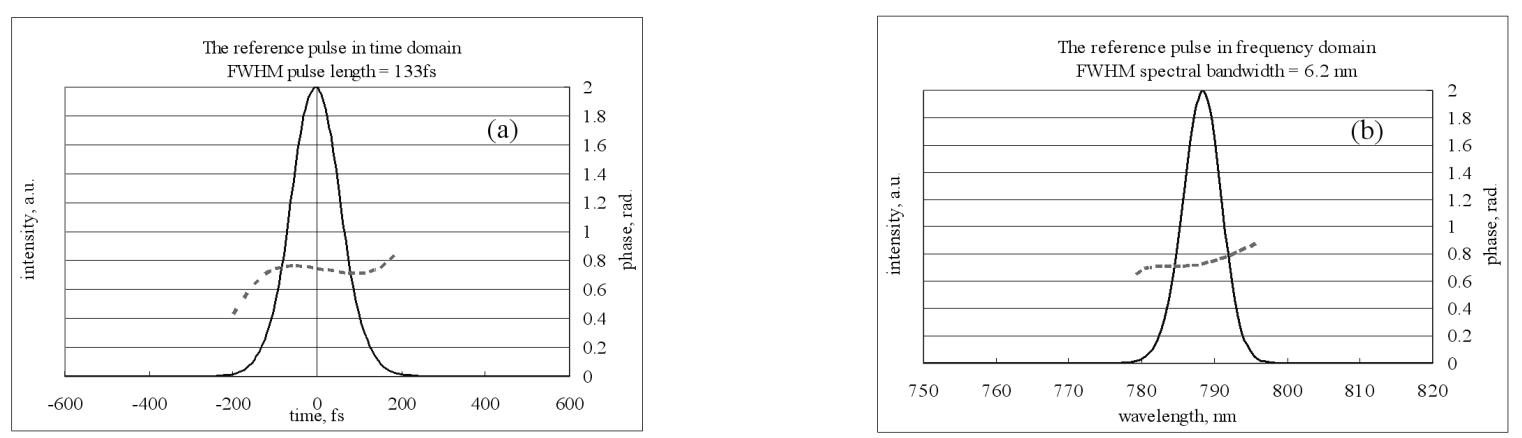

FIG. 3. (a) Temporal, and (b) spectral intensity and phase of the reference pulse.
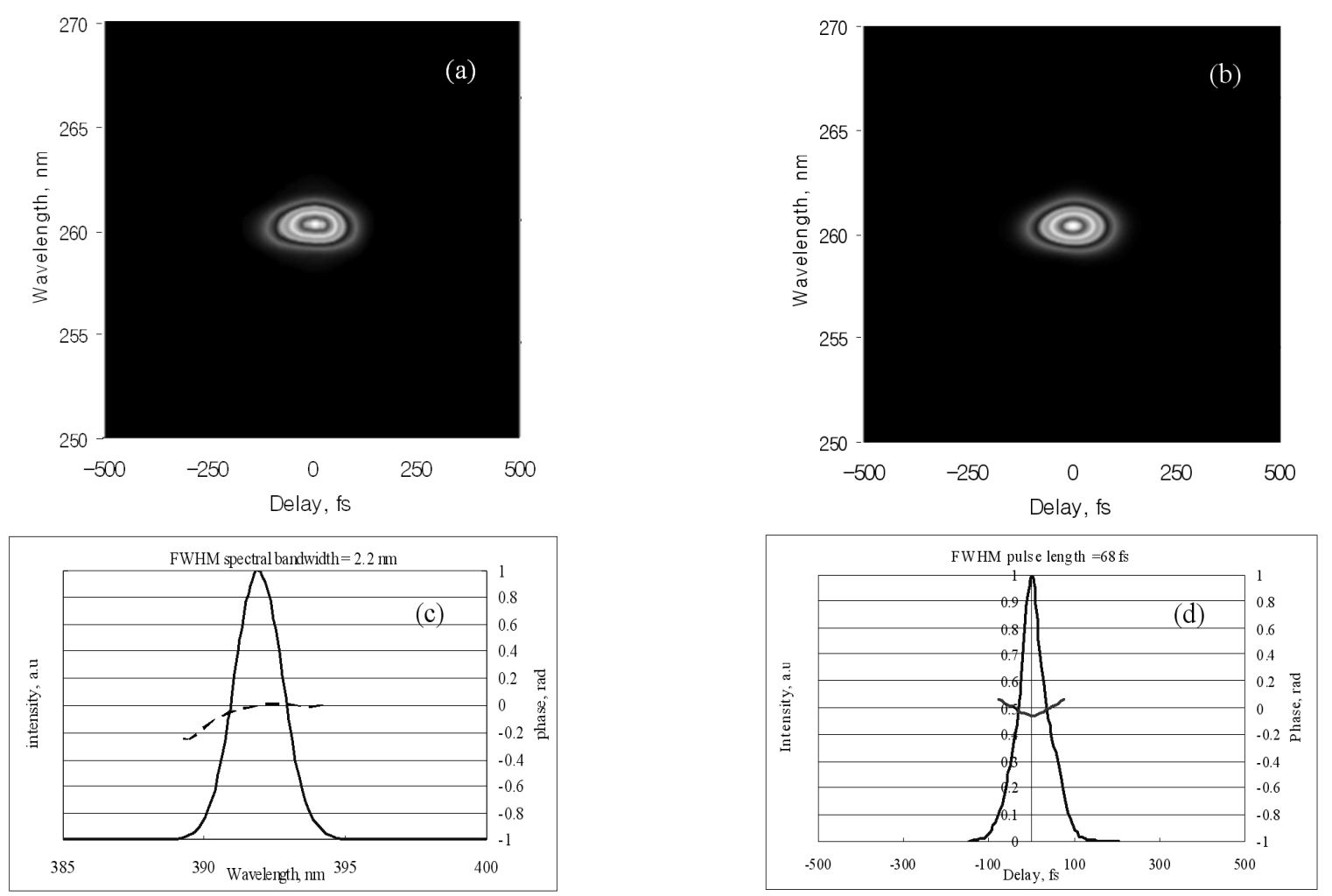

FIG. 4. SFG XFROG measurement of the UV, SH pulse: (a) measured XFROG trace, (b) retrieved XFROG trace, (c) retrieved spectrum (solid) and phase (dashed), (d) retrieved temporal intensity (solid) and phase (dashed). 
the phase retrieval algorithm.

From the trace, we have succeeded in retrieving the temporal and the spectral intensity as well as phase of the unknown $\mathrm{SH}$ pulses. The measured and the retrieved XFROG traces, and corresponding temporal and spectral characteristics of the retrieved UV pulses are shown in Fig. 4. The retrieved spectrogram fairly reproduces the measured one with an error $\mathrm{G}=0.0068$ [4]. The retrieved $\mathrm{SH}$ pulse shows a 68 fs temporal width and a $2.2 \mathrm{~nm}$ spectral bandwidth.

In order to check the consistency of the XFROG measurements, we have compared the retrieved spectral phase and spectrum of the pulses measured by using the XFROG technique without and with a 2-cm-thick BK7 glass rod on the path of the UV pulses. Assuming that the BK7 rod keeps the spectrum of the UV pulses unchanged and neglecting the third and higher order terms of the dispersion, we could estimate the intentional chirp of the pulse which was induced by a passage through the BK7 rod. Electric field of a pulse after passing through a medium with a length of $l$ can be written as

$$
E(\omega, l)=E(\omega, 0) e^{i \frac{b}{2}\left(\omega-\omega_{0}\right)^{2 l}}
$$

where $b$ is the coefficient of the group velocity dispersion (GVD) defined as

$$
b=\left.\frac{\lambda_{0}^{3}}{2 \pi c^{2}} \frac{d^{2} n}{d \lambda^{2}}\right|_{\lambda_{0}} .
$$

Where $n$ is the refractive index, $\mathrm{c}$ is the speed of light in vacuum, and $\lambda_{0}$ is the center wavelength of the pulse spectrum.

Fig. 5 shows XFROG traces of the pulses, which were measured without and with a 2 -cm-thick BK7 glass rod on the beam path. The latter trace shows a slight tilt which is due to the additional positive dispersion of the BK7 rod.

The retrieved spectra and spectral phases of the two
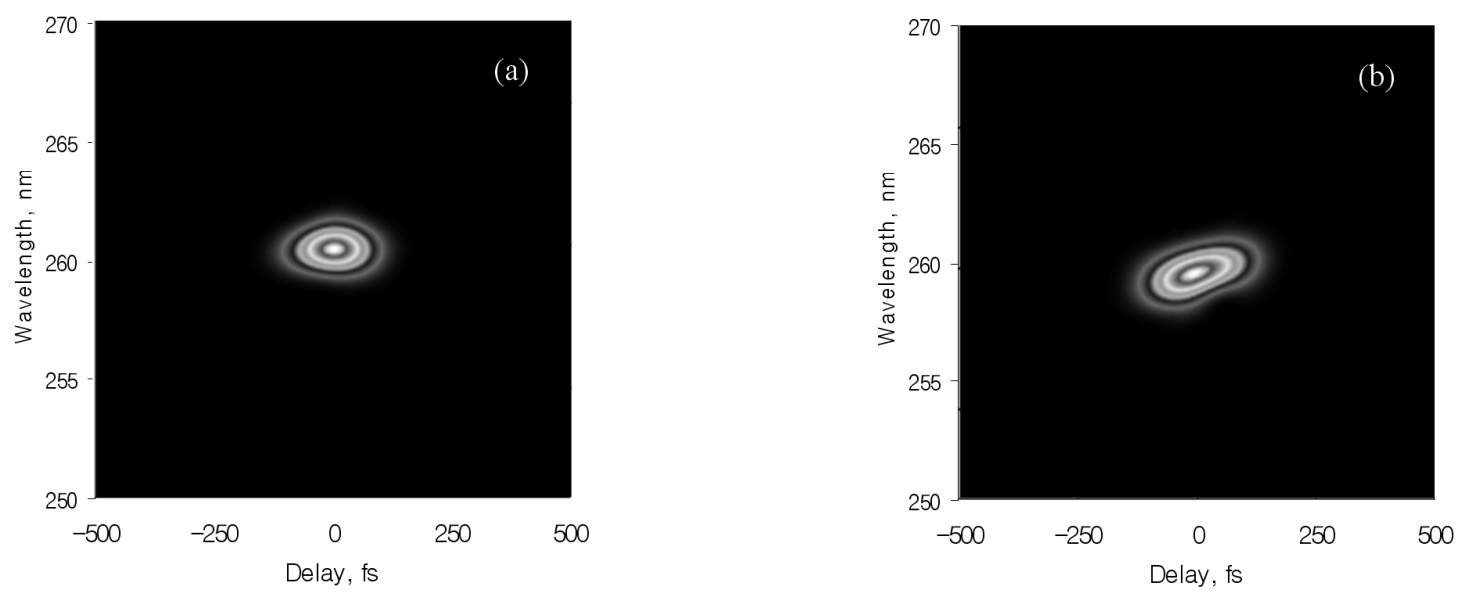

FIG. 5. (a) The retrieved XFROG trace (without a BK7 rod) (b) The retrieved XFROG trace (with a 2-cm-thick BK7 rod).

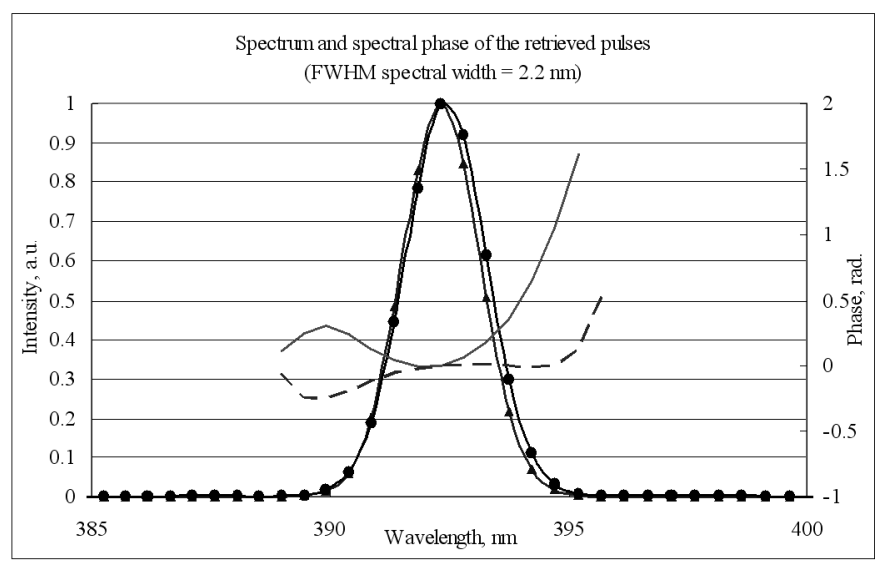

FIG. 6. The spectrum and spectral phase of the pulses measured without and with a 2-cm-thick BK7 glass rod on the beam path $(\boldsymbol{\Delta}$-spectrum, dashed line - spectral phase (without a BK7 rod), $\bullet$ - spectrum, solid line -spectral phase (with a 2-cm-thick BK7 rod)). 


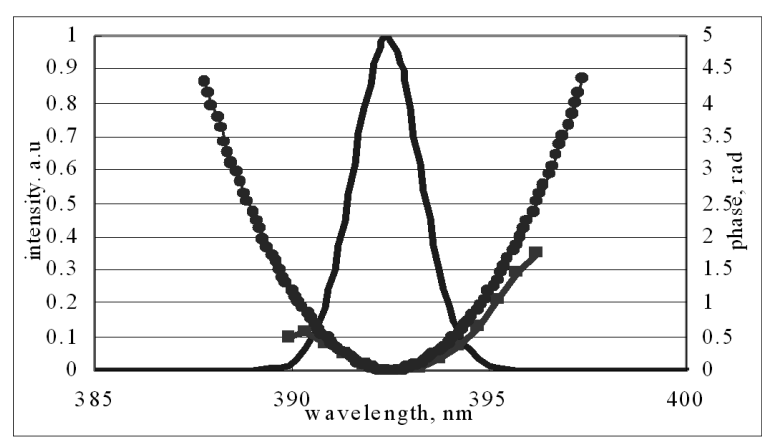

FIG. 7. The calculated and retrieved GVD of a 2-cmthick BK7 rod as a function of wavelength

(• - calculated, $\mathbf{\square}$ - retrieved ).

cases are shown in Fig. 6. The spectra of the two pulses are almost identical while spectral phases are significantly different.

Taking the difference between the retrieved spectral phases into account, we obtain the experimental value of the GVD coefficient of the BK7 $\operatorname{rod}\left(\mathrm{b}=1182 \pm 43 \mathrm{fs}^{2} / \mathrm{cm}\right)$, which is comparable to the theoretical value $\left(b=1250 \mathrm{fs}^{2} / \mathrm{cm}\right)$ calculated by using the Eq. 4 and the Sellmeier equation. Figure 7 shows the calculated and the measured GVD coefficients of the 2-cm-thick BK7 glass rod.

The agreement between the experimental and the theoretical results supports that the XFROG technique works well for measuring the intensity and the phase of the UV pulses.

\section{Supercontinuum pulse characterization}

Arguably, ultrabroadband supercontinuum is the most complex ultrashort pulse ever generated, which can be routinely generated using a PCF by injecting only nJ pulses from a Ti:sapphire oscillator. Many applications of the supercontinuum pulses require full information about their phases and intensities. But, the full characterization of the supercontinuum pulse meets some difficulties because of a demanding broad phasematching bandwidth, a large time-bandwidth-product and a weak intensity of the supercontinuum pulse. Fortunately, a SFG XFROG technique with the crystal dithering makes it possible to overcome above mentioned difficulties $[2,7]$.

The main challenge in attempting to use XFROG (or any other potential method) to measure the supercontinuum is obtaining sufficiently wide phase-matching bandwidth in the SFG crystal. In general, phase matching bandwidth of the nonlinear medium is inversely proportional to its thickness, and one can increase the phase matching bandwidth by using a sufficiently thin crystal. Due to a broad spectrum of the supercontinuum pulse, XFROG measurement typically demands an extremely thin, sub-ten micron crystal. However, the SFG signal is proportional to the square of the crystal thick- ness and a thin crystal generates a very weak signal resulting in a very low sensitivity.

P. O'Shea et al. proposed a novel idea that the phase matching bandwidth could be increased by using an angle-dithered, relatively thick, and hence narrowband crystal [13]. The key point was that the phase matching bandwidth doesn't need to exceed the pulse bandwidth for each pulse in a multi-shot measurement. Instead of this, it is required for the integrated phase matching bandwidth over a measurement time to exceed the pulse bandwidth. As a result, only a fraction of the pulse spectrum needs to be phase-matched at a shot as long as the remaining portions of the spectrum are phase matched at other shots during the signal integration time. Because the center wavelength of a phasematching band depends sensitively on the angle of incidence, one should let a SFG crystal angle-dither to yield a significantly increased effective phase-matching bandwidth for a given crystal thickness. As the SFG signal is proportional to the square of the crystal thickness, an angle-dithered, thick crystal yields significantly stronger signal. The crystal dithering technique has been successfully applied to the several pulse characterization techniques including FROG [14], XFROG [15] and SPIDER [16].

In order to achieve a phase-matching over the whole spectral bandwidth of the supercontinuum pulses, we have estimated appropriate dithering amplitude and the crystal's cut angle. For a given three-wave interaction, such as SFG, the exact phase matching angle can be determined using analytical expressions [17]. Suppose, we generate SFG signal using a BBO crystal with Type I phase matching condition. If we assume that the supercontinuum pulse's spectrum extends from $\lambda_{\min }=450 \mathrm{~nm}$ to $\lambda_{\max }=1200 \mathrm{~nm}$ and the reference pulse has a central wavelength of $\lambda_{\text {ref }}=800 \mathrm{~nm}$, corresponding exact phase matching angles for the SFG signal can be found as $40.75^{\circ}$ and $24.28^{\circ}$, respectively. As a result, the BBO crystal's cut angle has to be chosen around $32.5^{\circ}$ and the dithering amplitude should be at least $8.3^{0}$. In practice, however, we choose a larger amplitude of dithering than this.

The experiment for SFG XFROG has been performed in four steps [15,18-20]: Supercontinuum pulse generation, reference pulse characterization, XFROG measurement, and phase retrieval. A schematic diagram of the experimental setup is shown in Fig. 8.

The supercontinuum pulses were generated by launching output pulses from a Ti:sapphire laser system into a 4-cm-long PCF which has zero dispersion at 750 $\mathrm{nm}$ and a core size of $1.7 \mu \mathrm{m}$. The laser emitted 100 fs pulses at $780 \mathrm{~nm}$ central wavelength with energy of about $3 \mathrm{~nJ}$ at the rate of $82-\mathrm{MHz}$. The output pulses of the laser passed through a broadband optical isolator [21] which blocked the feedback of reflected light that may disturb the mode-locked operation of the laser 
oscillator. Then the pulse was divided into two pulses by a 50:50 beamsplitter. One part of the split pulses was used as reference for the SFG XFROG measurements while the other part traveled through a $40 \mathrm{X}$ microscope objective to be coupled into the 4-cm-long PCF for generation of the supercontinuum pulses. The supercontinuum pulses coming from the PCF were collimated by a 20X microscope objective and directed to a BBO crystal for sum frequency signal generation.

The intensity and the phase of the reference pulses were characterized by a simple SHG FROG apparatus. The experimental result showed that the temporal width at FWHM was about $100 \mathrm{fs}$ and the spectral bandwidth at FWHM was $10 \mathrm{~nm}$ for the reference pulse.

The completely characterized reference pulses were

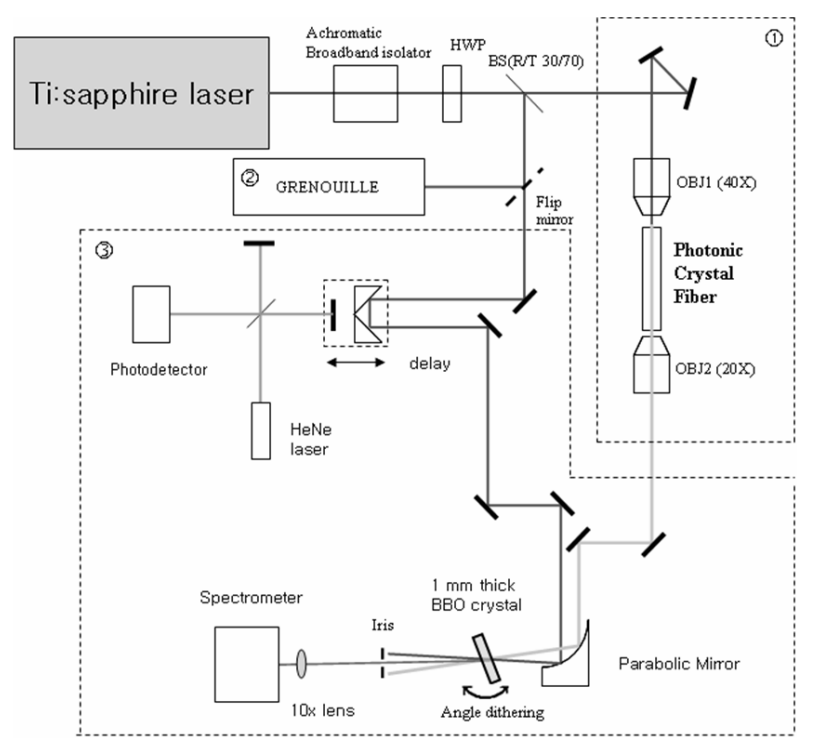

FIG. 8. Experimental setup for the supercontinuum pulse characterization by using a SFG XFROG.

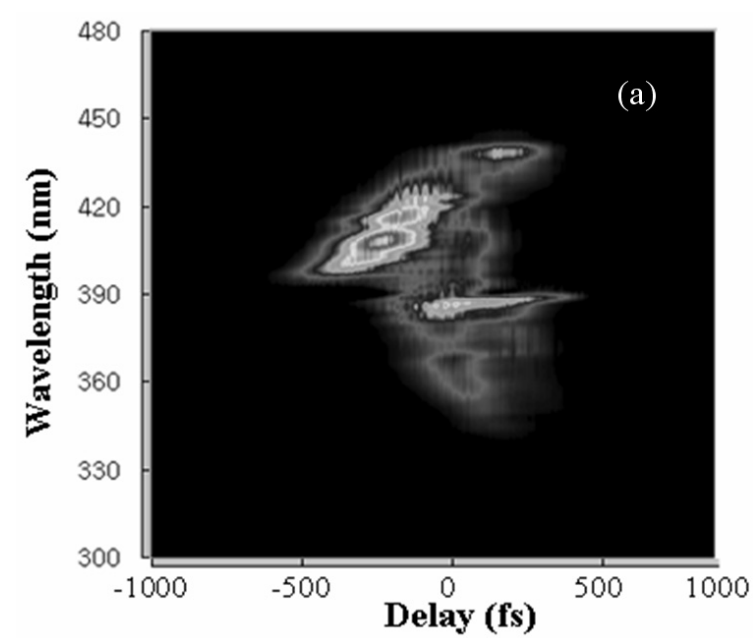

guided through a variable time delay so that they temporarily overlapped with the supercontinuum pulses. To generate the sum frequency signal, the reference and the unknown supercontinuum pulses were focused by an aluminum-coated, parabolic mirror $(\mathrm{f}=75 \mathrm{~mm})$ onto an 1-mm-thick BBO crystal, which was rapidly angle-dithering to achieve a phase matching over the whole bandwidth of the supercontinuum pulse. Angledithering of the crystal was performed by a galvanometer with amplitude of about $15^{0}$ and the dithering frequency was selected depending on the signal integration time of the spectrometer. We adopted a noncollinear geometry for SFG, and made the crossing angle of the pulses about 3 degrees. The relative delay between the supercontinuum and the reference pulses was controlled by a Michelson interferometer with an increment of $1.77 \mathrm{fs}$. The full spectrally-resolved measurements of the sum frequency signal at each delay step yielded a XFROG trace. Our measured raw trace had a grid size of $512 \times 1536$, and we further interpolated it into a size of $2048 \times 2048$ for the convenience of the phase retrieval.

In order to retrieve the intensity and phase of the supercontinuum pulse from the SFG XFROG trace, we have used an iterative Fourier transform algorithm with generalized projections [10]. The measured and the retrieved XFROG traces show good agreement, as can be seen in Fig. 9, and the major features of the measured trace have been successfully reproduced in the retrieved one with an error of $G=0.0522$. The FROG error $G$ is the measure for convergence of the phase retrieval and it's proportional to the normalized difference between the experimental and retrieved traces.

As shown in Fig. 10, we have plotted the retrieved intensity and the phase of the continuum pulse in the temporal and the spectral domain. Even though it shows a qualitative similarity between the measured and the

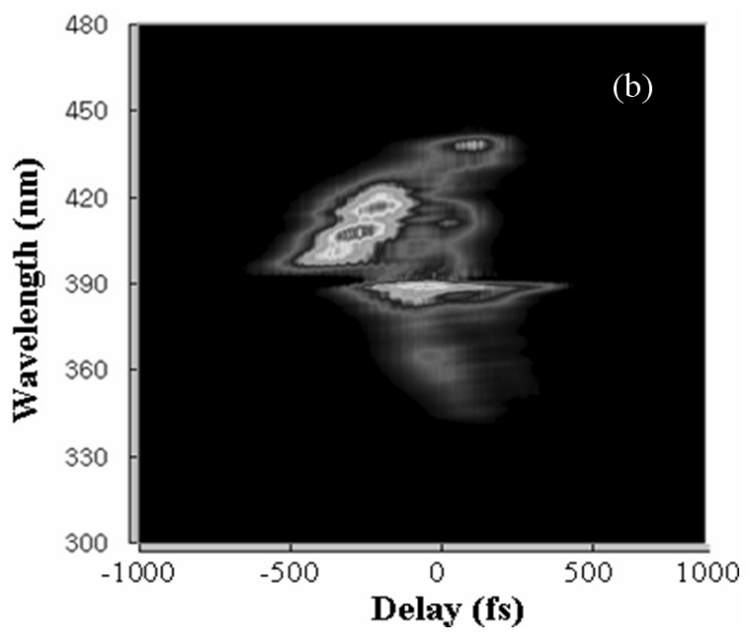

FIG. 9. (a) The measured XFROG trace, (b) the retrieved XFROG trace. 

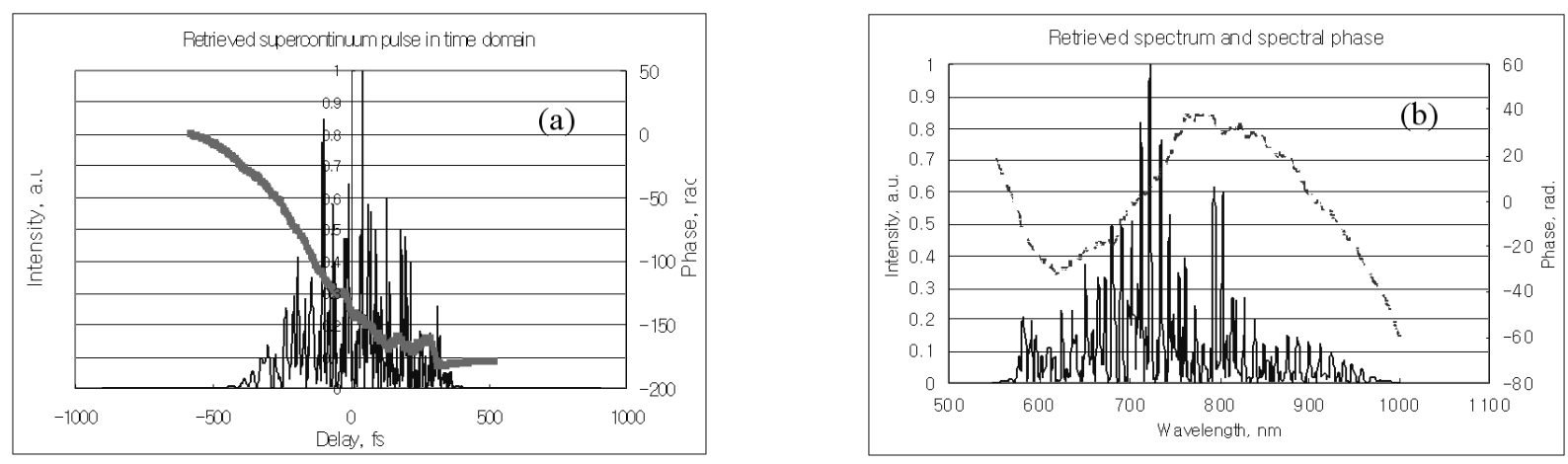

FIG. 10. (a) Temporal and, (b) spectral intensity and phase of the retrieved supercontinuum pulse.

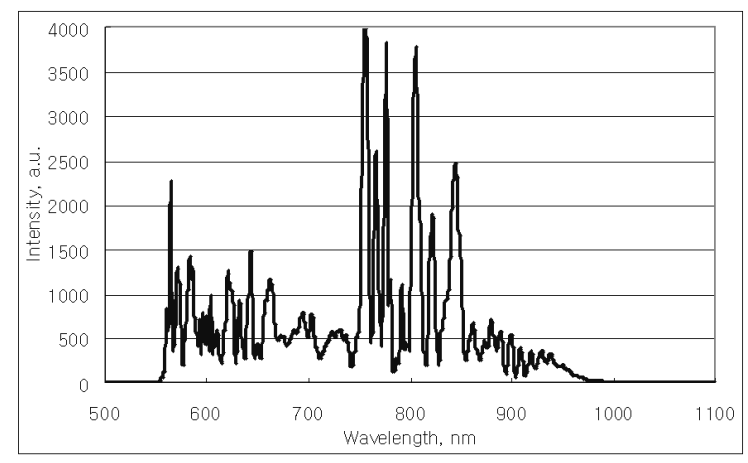

FIG. 11. Spectral intensity of the supercontinuum.

retrieved trace, the retrieved spectrum profile revealed fine structures which are absent in the spectrum of the supercontinuum measured independently by using a spectrometer (Fig. 11). Such disparity was already noted and explained as the result of shot-to-shot variation of supercontinuun because the nonlinear process involved is very sensitive to any slight change in environmental conditions $[4,15]$. But the overall shape, positions of main peaks and the bandwidth of the retrieved and the measured spectra were in a good agreement.

\section{CONCLUSIONS}

We have characterized the intensity and the phase of the supercontinuum, and the UV pulses by using a SFG XFROG technique.

Even though the supercontinuum pulse had quite complex structures in the temporal as well as the spectral domain, the retrieved spectrum agreed fairly well with an independently-measured spectrum. However, the retrieved spectrum has an incredible amount of fine structure that was not observed in the independently measured spectrum.

The difference of the frequencies between the reference and the unknown pulses make a XFROG technique more powerful to measure inconvenient wavelengths like the UV region. We have characterized the intensity and phase of the UV pulses generated from a frequency doubler.

In order to check the consistency of the XFROG measurement, we have compared the retrieved spectral phase and spectrum of the UV pulses which have been measured by XFROG technique without and with a 2-cm-thick BK7 glass rod on the beam path. The agreement between the theoretical and the retrieved GVD coefficients of the BK7 rod shows the reliability of the XFROG technique.

\section{ACKNOWLEDGMENTS}

This work is supported in part by a Korea Research Foundation grant (KRF-2006-005-J00302) and by the Nano-Bioelectronics and Systems Research Center (Seoul National University), an Engineering Research Center supported by the Korea Science and Engineering Foundation. Baatarchuluun Tsermaa was supported by the grant of Post-doctoral Program, Chonbuk National University (2009).

\section{REFERENCES}

1. G. D. Reid and K. Wynne, Ultrafast Laser Technology and Spectroscopy (Wiley, Chichester, 2000).

2. H. Kang, C. C. Byeon, M.-K. Oh, D.-K. Ko, J. Lee, J. S. Kim, H.-G. Choi, M. S. Jeong, and C.-S. Kee, "Coherent absorption spectroscopy with supercontinuum for semiconductor quantum well structure," J. Opt. Soc. Korea 11, 138-141 (2007).

3. I.-B. Shon, Y.-C. Noh, Y. S. Kim, D.-K. Ko, J. Lee, and Y.-J. Choi, "Laser ablation of polypropylene films using nanosecond, picosecond, and femtosecond laser," J. Opt. Soc. Korea 12, 38-41 (2008).

4. R. Trebino, Frequency-Resolved Optical Gating (Kluwer, Boston, 2002).

5. R. Trebino, K. W. DeLong, D. N. Fittinghoff, J. N. Sweetser, M. A. Krumbugel, B. A. Richman, and D. J. Kane, "Measuring ultrashort laser pulses in the 
time-frequency domain using frequency-resolved optical gating," Rev. of Sci. Inst. 68, 3277-3295 (1997).

6. R. Trebino and D. J. Kane, "Using phase retrieval to measure the intensity and phase of ultrashort pulses: frequency-resolved optical gating," J. Opt. Soc. Am. A 10, 1101-1111 (1993).

7. X. Gu, S. Akturk, A. Shreenath, Q. Cao, and R. Trebino, "The measurement of ultrashort light pulsessimple devices, complex pulses,” Opt. Rev. 11, 141-152 (2004).

8. C. Iaconis and I. Walmsley, "Self-referencing spectral interferometry for measuring ultrashort optical pulses," IEEE J. of Quantum Electron. 35, 501-509 (1999).

9. S. Linden, H. Giessen, and J. Kuhl, "XFROG: a new method for amplitude and phase characterization of weak ultrashort pulses," Phys. Stat. Sol. (b) 206, 119 124 (1998).

10. K. W. Delong, D. N. Fittinghoff, R. Trebino, B. Kohler, and K. Wilson, "Pulse retrieval in frequency-resolved optical gating based on the method of generalized projections," Opt. Lett. 19, 2152-2154 (1994).

11. P. O’Shea, M. Kimmel, X. Gu, and R. Trebino, "Highly simplified ultrashort pulse measurement," Opt. Lett. 26, 932-934 (2001).

12. B. K. Yang, H. S. Rho, J. M. Seo, and J. S. Kim, "Design, construction and calibration of a GRENOUILLE, single-shot, ultrashort-pulse measurement system," J. Korean Phys. Soc. 52, 269-274 (2008).

13. P. O'Shea, M. Kimmel, X. Gu, and R. Trebino, "Increasedbandwidth in ultrashort-pulse measurement using an angle-dithered nonlinear-optical crystal," Opt. Exp. 7,
342-349 (2000).

14. B. Tsermaa, B. K. Yang , J. M. Seo, and J. S. Kim, "A sub-10-fs Ti:sapphire oscillator with a simple fourmirror cavity,” J. Korean Phys. Soc. 52, 1043-1047 (2008).

15. J. M. Dudley, X. Gu, L. Xu, M. Kimmel, E. Zeek, P. O'Shea, R. Trebino, S. Coen, and R. Windeler, "Crosscorrelation frequency resolved optical gating analysis of broadband continuum generation in photonic crystal fiber: simulations and experiments," Opt. Exp. 10, 12151221 (2002).

16. B. Tsermaa, K. Myung-Whun, K. H. Lee, and J. S. Kim, "Sensitivity improvement of SPIDER by applying a crystal dithering technique," J. Korean Phys. Soc. 54, to be published (2009).

17. V. G. Dmitriev, G. G. Gurzadyan, and D. N. Nikogosyan, Handbook of Nonlinear Optical Crystals, $3^{\text {rd }}$ revised edition (Springer, Berlin, 1999).

18. Q. Cao, X. Gu, E. Zeek, M. Kimmel, R. Trebino, J. Dudley, and R. S. Windeler, "Measurement of the intensity and phase of supercontinuum from an 8-mm-long microstructure fiber,” Appl. Phys. B 77, 239-244 (2003).

19. X. Gu, L. Xu, M. Kimmel, E. Zeek, P. O’Shea, A. Shreenath, and R. Trebino, "Frequency-resolved optical gating and single-shot spectral measurements reveal fine structure in microstructure-fiber continuum," Opt. Lett. 27, 11741176 (2002).

20. Z. Zhu and T. G. Brown, "Experimental studies of polarization properties of supercontinua generated in a birefringent photonic crystal fiber,” Opt. Exp. 12, 791-796 (2004).

21. B. Tsermaa, J. S. Kim, and B. K. Yang, "Construction of a broad-band optical isolator by using an achromatic circular polarizer,” J. Korean Phys. Soc. 48, 892-896 (2006). 